\title{
Roadmap for double hypernuclei spectroscopy at PANDA
}

\author{
J. Pochodzalla ${ }^{1, a}$ and A. Sanchez Lorente ${ }^{1}$ on behalf of the PANDA collaboration \\ Institut für Kernphysik, Joh. Gutenberg-Univ. Mainz Germany.
}

\begin{abstract}
Hypernuclear Physics is currently attracting renewed attention. Thanks to the use of stored $\bar{p}$ beams, copious production of double $\Lambda$ hypernuclei is expected at the $\bar{P}$ anda experiment which will enable high precision $\gamma$-spectroscopy of such nuclei for the first time. In the present work we have studied the population of particle stable, excited states in double hypernuclei after the capture of a $\Xi^{-}$within a statistical decay model. In order to check the feasibility of producing and performing $\gamma$-spectroscopy of double hypernuclei at $\bar{P} A N D A$, an event generator based on these calculations has been implemented in the PANDA simulation framework PANDARоoт.
\end{abstract}

\section{Introduction}

The $\bar{P}$ anda experiment [2] which is planned at the international Facility for Antiproton and Ion Research FAIR in Darmstadt aims at the high resolution $\gamma$-ray spectroscopy of double hypernuclei $([1,3])$.

For that, excited states of $\Xi$ hypernucle will be used as a gateway to form double $\Lambda$ hypernuclei. The production of low momentum $\Xi^{-}$hyperons and their capture in atomic levels is therefore essential for the experiment. At $\overline{\mathrm{P}}$ ANDA the reactions $\bar{p}+p \rightarrow \Xi^{-} \bar{\Xi}^{+}$and $\bar{p}+n \rightarrow \Xi^{-} \bar{\Xi}^{0}$ followed by re-scattering of the $\Xi^{-}$within the primary target nucleus will be employed. After stopping the $\Xi^{-}$in an external secondary target, the formed $\Xi$ hypernuclei will be converted into double $\Lambda$ hypernuclei. The associated $\bar{\Xi}^{+}$ will undergo scattering or (in most cases) annihilation inside the residual nucleus and can be used as a tag for the reaction.

Because of this multi-stage process (see Fig. 1), spectroscopic studies, based on the analysis of two-body reaction kinematics like in single hypernuclei reactions, cannot be performed and spectroscopic information on double hypernuclei can only be obtained via their decay products:

- $\gamma$-rays emitted via the sequential decay of an excited double hypernucleus provide detailed information on the level structure.

- Once the ground-state is reached, pions and protons from the mesonic or non-mesonic weak decays can be used to tag the reaction.

- A complete detection of the decay products from the excited residue is in principle possible, though a resolution comparable to nuclear emulsion would be required. In addition, except for the case of very light hypernuclei also neutral particles are emitted which usually escape the detection. As a consequence, the determination of the ground-state mass of double hypernu-

\footnotetext{
${ }^{a}$ e-mail: pochodza@kph.uni-mainz.de
}

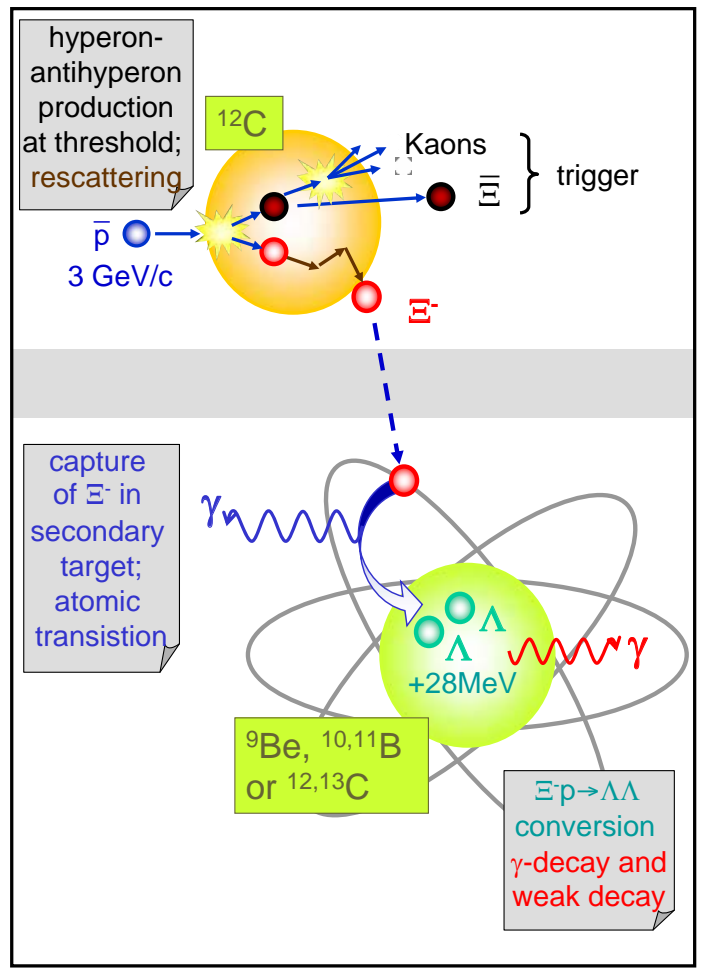

Fig. 1. Various steps of the double hypernucleus production in PANDA.

clei is limited to those light nuclei which decay exclusively into charged particles.

Therefore, a unique identification of the double hypernuclei can only be reached via the emitted $\gamma$-rays from excited, particle stable states[3]. In the present work we explore the feasibility to perform $\gamma$-spectroscopy of double hypernuclei at the planned $\bar{P} A N D A$ experiment. 


\section{Population of Excited States in Double Hypernuclei}

In order to limit the number of possible transitions and thus to increase the possible signal to background ratio, we focus in the following on light nuclei with mass numbers $A_{0} \leq 13$, where even a relatively small excitation energy may be comparable to their binding energy. In the following we therefore assume that the principal mechanism of de-excitation is the explosive decay of the excited nucleus into several smaller clusters. To describe this break-up process and in order to estimate the population of individual excited states in double hypernuclei after the conversion of the $\Xi^{-}$, we have developed a statistical decay model which is reminiscent of the Fermi break-up model $([4,5])$. We assume that the nucleus decays simultaneously into cold or slightly excited fragments([6]). In the case of conventional nuclear fragments, we adopt their experimental masses in ground states, and take into account their particle-stable excited states. For single hypernuclei, we use the experimental masses and all known excited states. For double hypernuclei we apply theoretically predicted masses and excited states $([7,8])$.

In the model we consider all possible break-up channels, which satisfy the mass number, hyperon number (i.e. strangeness), charge, energy and momenta conservations, and take into account the competition between these channels. Since the excitation energy of the initially produced double hypernuclei is not exactly known, we performed the calculations as a function of the binding energy of the captured $\Xi^{-}$. Calculations were performed for several stable secondary targets $\left({ }^{9} \mathrm{Be},{ }^{10} \mathrm{~B},{ }^{11} \mathrm{~B},{ }^{12} \mathrm{C}\right.$, and $\left.{ }^{13} \mathrm{C}\right)$ which lead to the production of excited states in double hypernuclei.

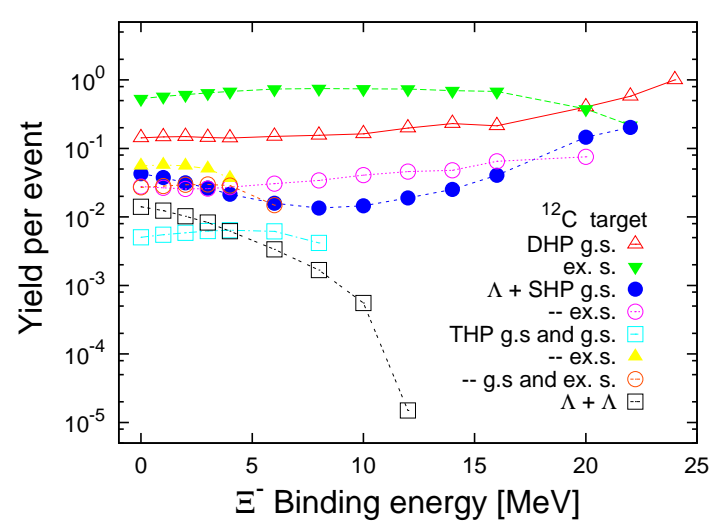

Fig. 2. Predicted relative yield for ground states (g.s.) and excited states (ex.s.) in double ( $\mathrm{DH})$, single ( $\mathrm{SH})$, and twin hypernuclei (TH) as a function of the $\Xi$ binding energy for a secondary ${ }^{12} \mathrm{C}$ target.

Fig. 2 shows as an example the production of ground (g.s.) and excited (ex.s.) states of conventional nuclear fragments as well as single (SHP), twin (THP) and double (DHP) hypernuclei in case of a ${ }^{12} \mathrm{C}$ target as a function of the assumed $\Xi^{-}$binding energy. According to these calculations excited states in double hypernuclei (triangles) are produced with significant probability. Fig. 3 shows the

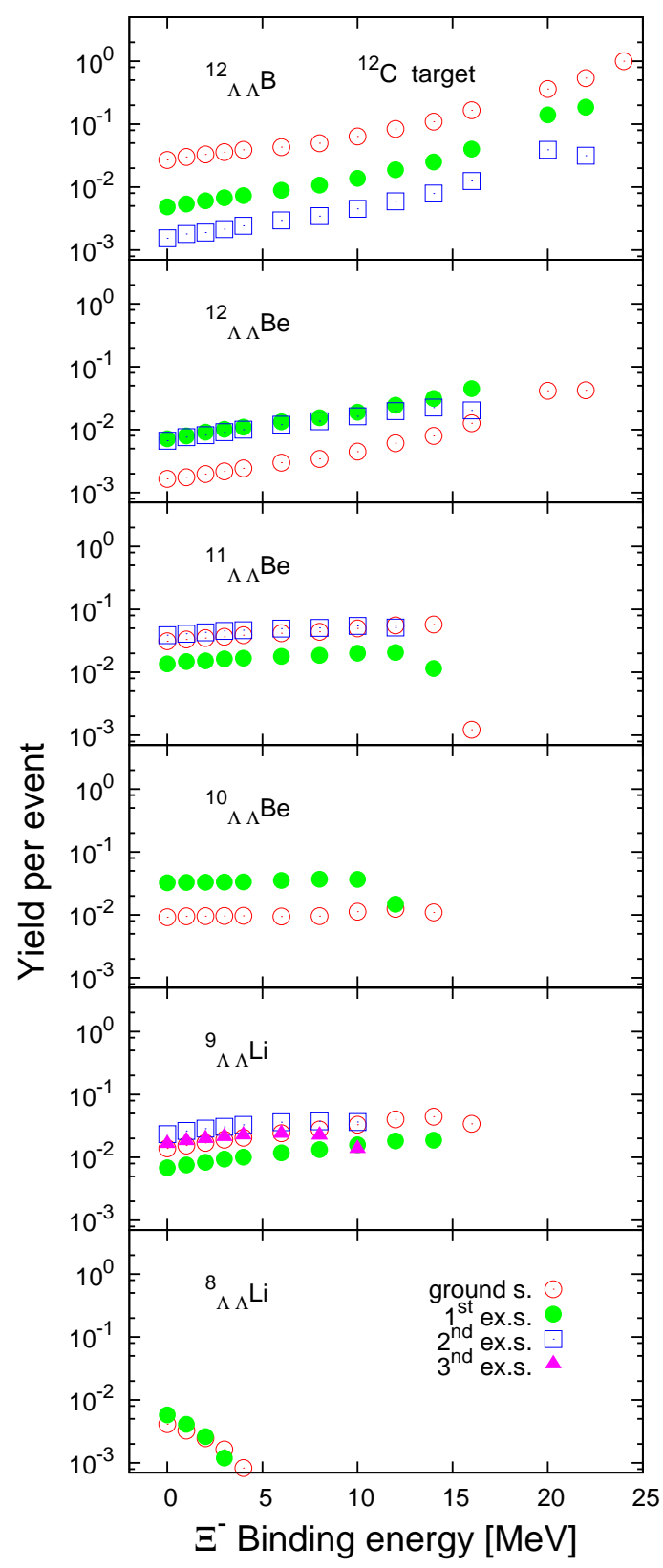

Fig. 3. Production probability of ground and excited states of accessible double hypernuclei after the capture of a $\Xi^{-}$in a ${ }^{12} \mathrm{C}$ nucleus and the $\Xi^{-}$conversion into two $\Lambda$ hyperons. Excited states in ${ }_{\Lambda \Lambda}^{11} \mathrm{Be},{ }_{\Lambda \Lambda}^{10} \mathrm{Be}$ and ${ }_{\Lambda \Lambda}^{9} \mathrm{Li}$ dominate over a wide range of the $\Xi^{-}$ binding energy.

population of the different accessible double hypernuclei For the ${ }^{12} \mathrm{C}$ target, excited states in ${ }_{\Lambda \Lambda}^{11} \mathrm{Be},{ }_{\Lambda \Lambda}^{10} \mathrm{Be}$ and ${ }_{\Lambda \Lambda}^{9} \mathrm{Li}$ dominate over a wide range of the assumed $\Xi^{-}$binding energy. 
$19^{\text {th }}$ International IUPAP Conference on Few-Body Problems in Physics

\section{Experimental Integration and Simulation}

The hypernuclei study will make use of the modular structure of PANDA. Removing the backward end-cap calorimeter will allow to add a dedicated nuclear target station and the required additional detectors for $\gamma$ spectroscopy (see 4 and ref.([3]))close to the entrance of $\bar{P}$ ANDA. While the detection of anti-hyperons and low momentum $\mathrm{K}^{+}$can be ensured by the universal detector and its PID system, a specific target system and a $\gamma$-detector are additional components required for the hypernuclear studies.

The two-step production mechanism and a devoted experimental setup has been implemented into the simulation package PANDARoot as well as the $\bar{P}$ ANDA setup.

- A primary carbon target at the entrance to the central tracking detector of PANDA.

- A small secondary active sandwich target composed of silicon detectors and ${ }^{9} \mathrm{Be},{ }^{10,11} \mathrm{~B}$ or ${ }^{12,13} \mathrm{C}$ absorbers to slow down and stop the $\Xi^{-}$and to identify the weak decay products.

- To detect the $\gamma$-rays from the excited double hypernuclei an array of 15 n-type Germanium triple Clusterarrays will be added. To maximize the detection efficiency the $\gamma$-detectors must be arranged as close as possible to the target at backward axial angles.

\section{Spectroscopic studies of double hypernuclei}

For the first step, namely the reaction $\bar{p}+p \rightarrow \Xi^{-} \bar{\Xi}^{+}$we have employed an event generator ([9]) which is based on an Intra Nuclear Cascade model and which takes as a main ingredient the rescattering of the antihyperons and hyperons in the target nucleus into account. From 50505 produced events which contained a $\Xi^{-}$with a laboratory momentum less than $500 \mathrm{MeV} / \mathrm{c}, 7396$ hyperons are stopped within the secondary target.

In the next step the excited particle stable states of double hypernuclei as well as excited states of conventional nuclei and single hypernuclei produced during the decay process de-excite via $\gamma$-ray emission. For the high resolution spectroscopy of excited hypernuclear states a Germanium $\gamma$-array detector([10]) has also been implemented in the standard PANDA framework PANDARoot ([3]). Fig. 5 shows the total energy spectrum summed over all germanium detectors for all events where a $\Xi^{-}$has been stopped in the secondary carbon target. Note, that the size of the bins $(50 \mathrm{keV})$ in this plot is significantly larger than the resolution of the germanium detectors expected even for high data rates at normal conditions $(3.4 \mathrm{keV}$ at $110 \mathrm{kHz}([11]))$. Several peaks seen in the spectrum around $1,1.68$ and $3 \mathrm{MeV}$ are associated with $\gamma$-transitions in various hypernuclei. However, for a clear assignment of these lines obviously additional experimental information will be needed.

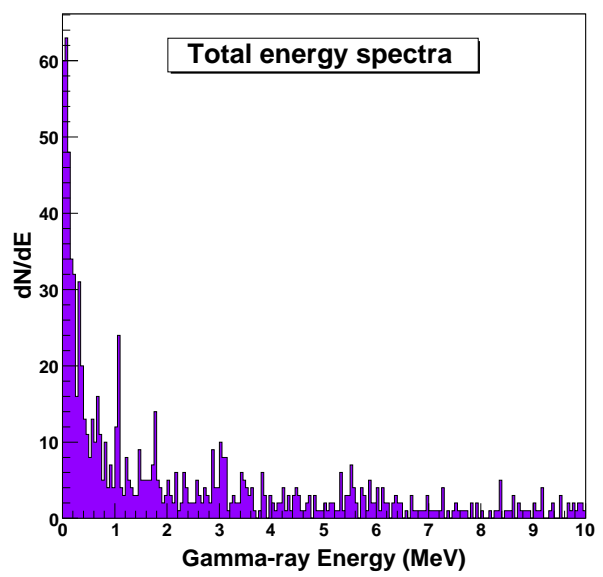

Fig. 5. Total $\gamma$-ray spectrum resulting from the decay of double hypernuclei produced in a ${ }^{12} \mathrm{C}$ target and detected in the germanium array and before additional cuts.

\section{Weak decays of Hypernuclei}

For the light hypernuclei to be studied in the initial phase of the planned experiments the non-mesonic and mesonic decays are of similar importance. In the following we will focus on the case of two subsequent mesonic weak decays of the produced double and single hypernuclei. For the light nuclei discussed below this amounts to about $10 \%$ of the total decay probability. Since the momenta of the two pions are strongly correlated their coincident measurement provides an effective method to tag the production of a double hypernucleus. Moreover, the momenta of the two pions are a fingerprint of the hypernucleus respective its binding energy.

The upper part of Fig. 6 shows the momentum correlation of all negative pion candidates from the secondary ${ }^{12} C$ target. The various bumps correspond to different double or twin hypernuclei. The good separation of the different double or twin hypernuclei provides an efficient selection criterion for their decays.

The lower part of Fig. 6 shows the $\gamma$-ray spectrum gated on each of four regions indicated in the two-dimensional scatter plot. In the plots (a) and (d) the $1.684 \mathrm{MeV}_{1^{2}}{ }^{+}$and the $2.86 \mathrm{MeV} 2^{+}$states of ${ }_{\Lambda \Lambda}^{11} \mathrm{Be}$ and ${ }_{\Lambda \Lambda}^{10} \mathrm{Be}$, respectively, can clearly be identified. Because of the limited statistics in the present simulations and the decreasing photopeak efficiency at high photon energies, the strongly populated high lying states in ${ }_{\Lambda \Lambda}^{9} \mathrm{Li}$ at 4.55 and $5.96 \mathrm{MeV}$ cannot be identified in (b). The two dominant peaks seen in part (c) result from the decays of excited single hyperfragments produced in the $\Xi^{-}+C \rightarrow{ }_{\Lambda}^{4} H+{ }_{\Lambda}^{9} B e$ reaction, i.e. ${ }_{\Lambda}^{4} H$ at an excitation energy of $1.08 \mathrm{MeV}([12,13])$ and ${ }_{\Lambda}^{9} B e$ at an excitation energy of 3.029 and $3.060 \mathrm{MeV}([14,15])$ are also well identified ( See ref. [3] for more details). The spectrum shown in Fig. 6 corresponds to a running time at PANDA of the order of two weeks. It is also important to realize that gating on double non-mesonic weak decays or on mixed weak decays may significantly improve the final rate. 

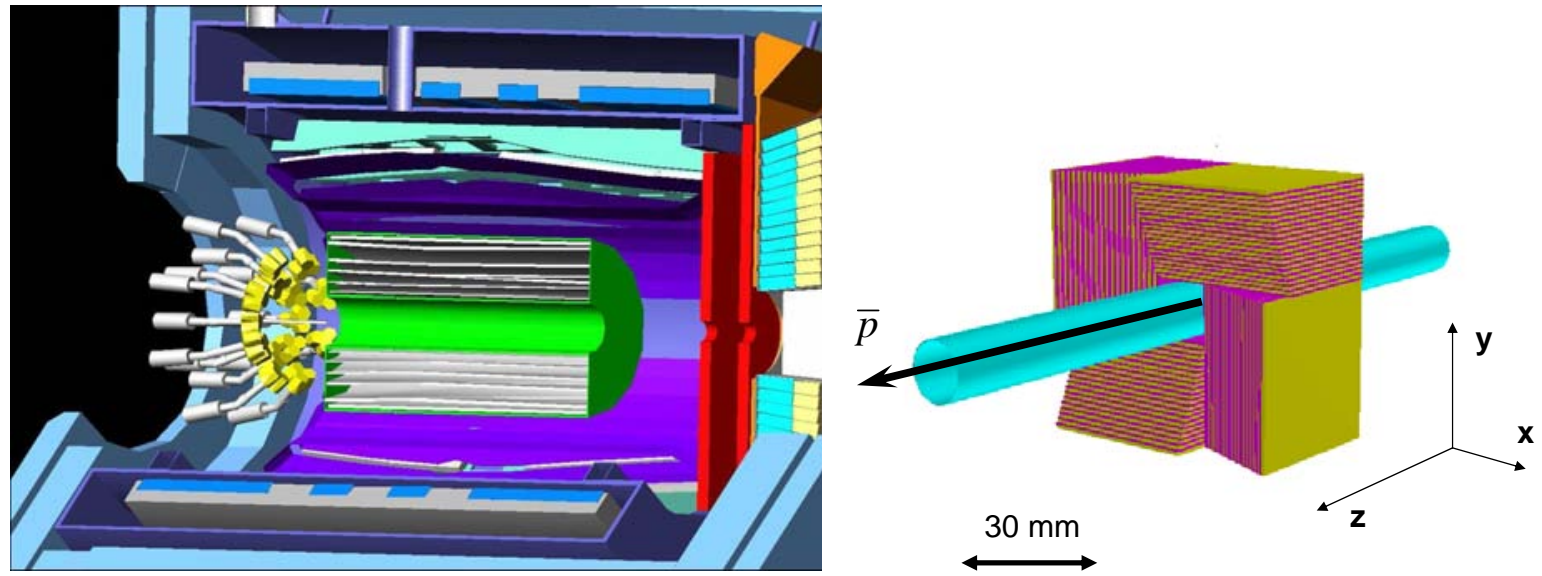

Fig. 4. Schematic view of the hypernucleus setup in $\bar{P}$ ANDA.
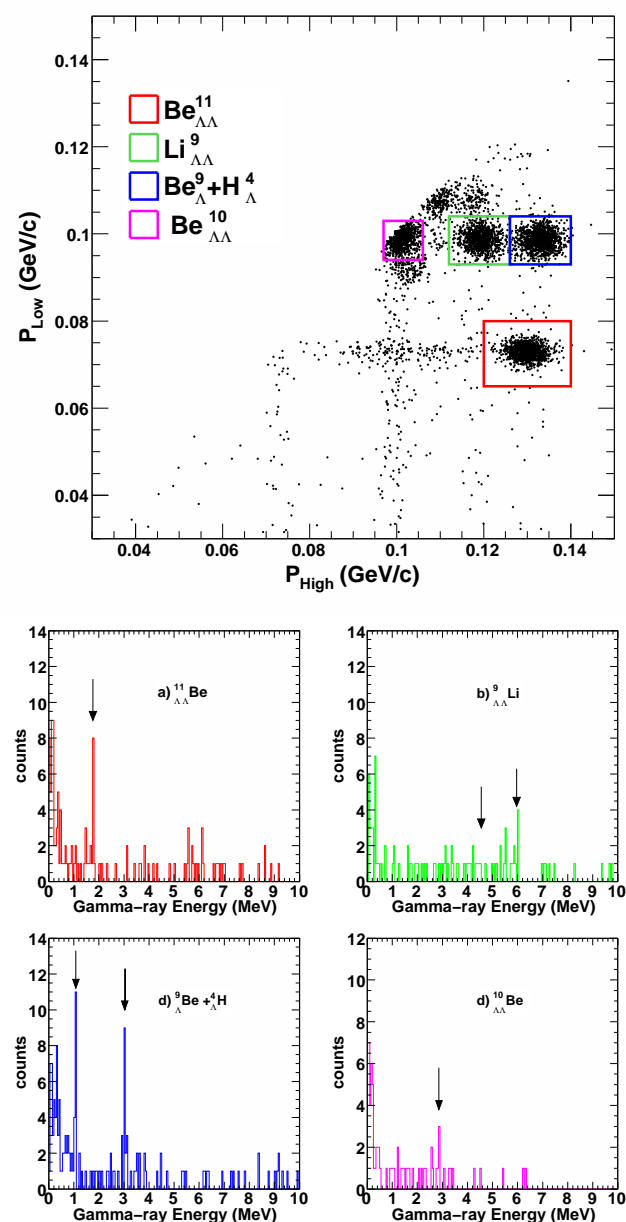

Fig. 6. Upper part: Momentum correlation of all negative pion candidates resulting from the decay of double hypernuclei in a secondary ${ }^{12} \mathrm{C}$ target. Lower part: $\gamma$-spectrum detected in the Ge-array by cutting on the two pion momenta. The expected $\gamma$-transitions energies from single and double hypernuclei are marked by the arrows.

\section{Background}

Particles produced simultaneously with the double hypernuclei do not significantly disturb the $\gamma$-ray detection.
The main limitation is the load of the Cluster-array by the high particles rate from uncorrelated background reactions. The $\bar{p} p \rightarrow \Xi^{-} \bar{\Xi}$ cross section of $2 \mu \mathrm{b}$ is about a factor 2500 smaller than the inelastic $p \bar{p}$ cross section of $50 \mathrm{mb}$ at $3 \mathrm{GeV} / \mathrm{c}$. The total energy spectra in the crystal has been obtained summing up event by event the energy contributions of the particles impinging on the Ge array. Background reactions have been calculated by using the UrQMD+SMM([16]) event Generator. For the present analysis $10000 \bar{p}+{ }^{12} C$ interactions at $3 \mathrm{GeV} / \mathrm{c}$ were generated([17]). The total energy spectra resulting from the background simulation have been filtered by using the same technique as it was done for the signal events and by applying identical cuts. For ${ }_{\Lambda \Lambda}^{11} \mathrm{Be}$ as well as ${ }_{\Lambda \Lambda}^{10} \mathrm{Be}$ only one single event survived the cuts. Both of these events had an energy deposition in the germanium detector exceeding $10 \mathrm{MeV}$ significantly.

Several further improvements of the background suppression are expected by exploring the topology of the sequential weak decays. This includes the analysis of tracks not pointing to the primary target, multiplicity jumps in the detector planes and the energy deposition in the secondary target. Furthermore kaons detected in the central detector of $\bar{P}$ ANDA at forward angles can be used to tag the $\bar{\Xi}$ production.

This research is part of the EU integrated infrastructure initiative Hadron- Physics Project under contract number RII3-CT-2004-506078. We acknowledge financial support from the Bundesministerium für Bildung und Forschung $(\mathrm{bmb}+\mathrm{f})$ under contract number 06MZ225I.

\section{References}

1. J. Pochodzalla, Nucl. Phys. A 754, 430c 2005.

2. Technical Progress Report for PANDA, http://www-panda.gsi.de/ db/papersDB/PC19050217_panda_tpr.pdf

3. Physics Performance Report for PANDA: Strong Interaction Studies with Antiprotons, arXiv:0903.3905v1.

4. E. Fermi, Progr. Theor. Phys. 5, 570 (1950). 
$19^{\text {th }}$ International IUPAP Conference on Few-Body Problems in Physics

5. A. Sanchez Lorente, A. Botvina and J. Pochodzalla, Production of hypernuclei via Fermi breakup of excited strange systems, (in preparation).

6. J.P. Bondorf et al., Phys. Rep. 257, 133 (1995).

7. E. Hiyama et al., Phys. Rev. 66, 024007 (2002).

8. T. Yamada and K. Ikeda, Phys. Rev. C56, 3216 (1997).

9. F. Ferroet al., Nucl. Phys. A789, 209 (2007).

10. A. Sanchez Lorente et al., Nucl. Inst. Meth A 573, 410 (2007)

11. M. Kavatsyuk et al., Performance of Germanium detectors at high counting rates, GSI Sci. Rep. 2007.

12. A. Bamberger et al., Nucl. Phys. B60, 1 (1973).

13. M. Bedjidian et al., Phys. Lett. 62, 467 (1976).

14. M. May et al., Phys. Rev. Lett.51, 2085 (1983).

15. H. Akikawa et al., Phys. Rev. Lett.88, 082501-1 (2002)

16. A. Galoyan, private comunication.

17. A. Sanchez Lorente et al., Particle Background simulation for a triple Germanium Cluster detectors at PANDA, in GSI Sci. Rep. 2007. 